\title{
Balance y simetría de la fuerza isométrica del hombro en nadadores del Club Flamingo de natación de la ciudad de Pereira
}

\author{
Jenny Paola Correa Ospina'; María Camila Cardona Santa'; \\ Alejandro Gómez Rodas ${ }^{2}$
}

RESUMEN Introducción: la natación es una actividad que genera una alta prevalencia de lesiones de hombro, debido a la cantidad de movimientos repetitivos y a la ausencia de tiempo de descanso; la debilidad, la posición anormal, y la sincronización anormal de los músculos tanto del hombro como de la cintura escapular, han sido asociados a disfunciones del movimiento que pueden conducir a alteraciones funcionales, finalización de la participación deportiva y discapacidad, por esto se hace necesario indagar sobre el balance de la fuerza isométrica del hombro en nadadores del Club Flamingo de natación de la ciudad de Pereira.

Método: estudio no experimental-descriptivo, de tipo empírico analítico y de carácter observacional analítico; la población de la investigación se constituyó por 19 nadadores pertenecientes al Club Flamingo de natación de la ciudad de Pereira de las categorías juvenil 1-2 (13 -17 años) y mayores (18 años en adelante). Se realizó un análisis univariado descriptivo con una segunda fase inferencial para determinar diferencias por género, tanto en la fuerza muscular de los músculos del hombro como por su dominancia lateral.

Resultados: se encontró una media para la edad en hombres y mujeres de 18 $\pm 4,1$ y $17,1 \pm 4,3$ años respectivamente, un índice de masa corporal de 22,8 $\pm 2 \mathrm{Kg} / \mathrm{m}^{2}$ y $22,2 \pm 1,1, \mathrm{Kg} / \mathrm{m}^{2}$ en hombres y en mujeres, con un porcentaje graso de $18,2 \% \pm 6,2$ y $29,9 \% \pm 3,2$ tanto en hombres como en mujeres. No se encontraron diferencias significativas en la fuerza muscular isométrica del hombro entre hombres y mujeres, como tampoco entre miembro superior dominante y no dominante $(\mathrm{p}>0,05)$. La fuerza muscular isométrica de cada músculo del hombro conserva un patrón especifico similar a lo encontrado en otros estudios.

Conclusión: en esta muestra de nadadores no existen diferencias en la fuerza muscular isométrica del hombro por género y dominancia lateral. Se observó un patrón de fuerza muscular similar a lo encontrado en otros estudios.

CLAVE Contracción Isométrica,

Nadadores

1 Estudiantes de séptimo semestre de Fisioterapia. Fundación Universitaria del Área Andina. Seccional Pereira. Integrante del semillero SEMFIS.

2 Docente Fundación Universitaria del Área Andina y Universidad Tecnológica de Pereira, Programas de Fisioterapia y Ciencias del Deporte y la Recreación 


\section{Balance and symmetry of the isometric strength of the shoulder in swimmers of the Flamingo Swimming Club of the city of Pereira}

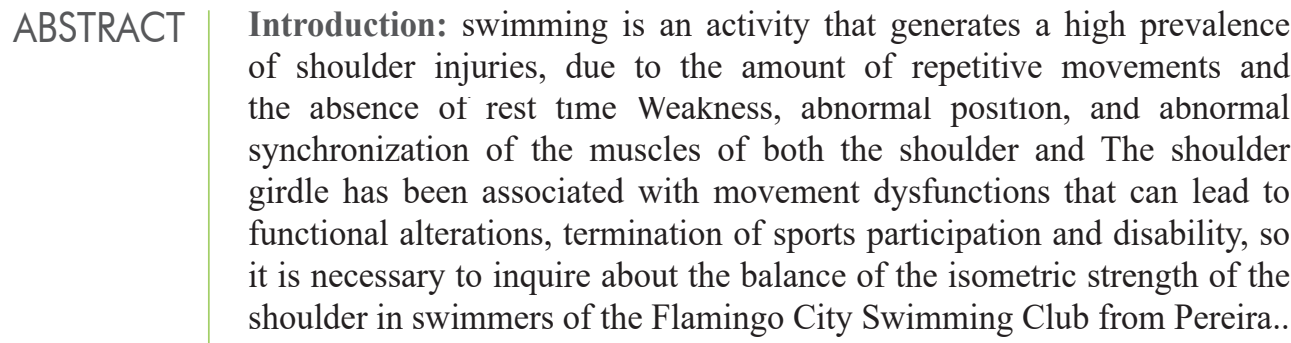

Method: non-experimental, descriptive, empirical analytical and observational analytical study; The research population consisted of 19 swimmers belonging to the Flamingo Swimming Club of the city of Pereira of the youth categories 1-2 (13-17 years) and older (18 years and older). A descriptive univariate analysis was performed with a second inferential phase to determine gender differences, both in the muscular strength of the shoulder muscles and their lateral dominance.

Results: a mean for age was found in men and women of $18 \pm 4.1$ and 17.1 \pm 4.3 years respectively, a body mass index of $22.8 \pm 2 \mathrm{Kg} / \mathrm{m} 2$ and $22.2 \pm$ $1,1, \mathrm{Kg} / \mathrm{m} 2$ in men and women, with a fat percentage of $18.2 \% \pm 6.2$ and $29.9 \% \pm 3.2$ in both men and women. There were no significant differences in isometric muscle strength of the shoulder between men and women, nor between dominant and non-dominant upper limb ( $p>0.05)$. The isometric muscle strength of each shoulder muscle retains a specific pattern similar to that found in other studies.

Conclusion: in this sample of swimmers there are no differences in isometric muscle strength of the shoulder by gender and lateral dominance. A muscle strength pattern similar to that found in other studies was observed.

Shoulder Injury,

Balance Dysfunction,

Isometric Contraction,

Swimmers 


\section{INTRODUCCIÓN}

La natación es una actividad que requiere primariamente de la parte superior del cuerpo para generar su fuerza propulsiva. Durante el avance del cuerpo en el agua, el hombro sufre una alta carga debido a la cantidad de movimientos repetitivos y la ausencia de tiempo de descanso (1), esto lleva a que se genere una alta prevalencia de lesión de hombro en este deporte, la cual se encuentra entre el 40\% y el 91\% (2).

La debilidad, la posición anormal, y la sincronización anormal de los músculos tanto del hombro como de la cintura escapular son todos factores que contribuyen a la discinesia escapular(3), por tanto es esencial, que los nadadores tengan una equilibrada función de su cintura escapular y un balance en la fuerza entre los grupos musculares agonistas y antagonistas del hombro (4), dado que un imbalance y asimetría en el control adecuado del complejo del hombro se ha asociado a deficiencias en el movimiento, provocando problemas tales como tensiones anormales en las estructuras capsulares anteriores del hombro, un mayor riesgo de lesiones del manguito rotador y disminución del rendimiento muscular. Además, las discinesias escapulares influyen en la posición de la articulación del hombro, disminuyendo el espacio subacromial, lo que lleva a pinzamientos subacromiales (3), que pueden conducir a alteraciones funcionales, finalización de la participación deportiva y discapacidad (5).

La alta asistencia de las personas tanto en deporte recreativo como competitivo en la natación clásica y la alta probabilidad de lesión de hombro por su práctica, hace necesario el conocimiento de factores de riesgo músculo-esqueléticos que pueden desencadenar lesiones, disminución del rendimiento deportivo y detrimento de la calidad de vida de los nadadores. Por tanto, el objetivo de la presente investigación fue determinar el balance y simetría de la fuerza muscular isométrica del hombro en nadadores del Club Flamingo de natación de la ciudad de Pereira.

\section{MATERIALES Y MÉTODOS}

Estudio no experimental-descriptivo, de tipo empírico analítico y de carácter observacional analítico, que incluyó una muestra no probabilística intencionada de 19 nadadores del Club Flamingo de natación de la ciudad de Pereira (9 del género femenino y 9 del género masculino), de las categorías juvenil 1-2 (13-17 años) y mayores (18 años en adelante). Se incluyeron nadadores activos con mínimo 6 meses de entrenamiento previo y se excluyeron aquellos con presencia de patología del hombro, antecedentes quirúrgicos o procedimientos ortopédicos que impidieran la evaluación de la fuerza isométrica del hombro. Las valoraciones fueron realizadas con firma previa de consentimiento informado, ajustándose a los criterios de la declaración de Helsinki.

La medición de la fuerza isométrica del hombro se llevó a cabo por un Fisioterapeuta experimentado, de acuerdo con los lineamientos descritos por Hislop y Montgomery (6) con un dinamómetro manual digital producido por Hoggan Scientific denominado microFET ${ }^{\circledR} 2$, el cual registra la medición de la fuerza en Newtons. La valoración isométrica con dinamómetro manual ha mostrado altos coeficientes de confiabilidad que oscilan entre 0,894 y 0,692 (3). Estas evaluaciones se llevaron a cabo en las 
instalaciones de la Liga de Risaralda de Natación en recinto cerrado y se efectuaron 3 mediciones de la fuerza muscular de los músculos trapecio superior, medio e inferior, así como del serrato anterior, romboides, rotadores externos y rotadores internos del miembro superior dominante y no dominante. Posteriormente se utilizó la media de estas evaluaciones para análisis de datos.

Los datos arrojados por las pruebas de la fuerza muscular isométrica fueron analizados en el programa estadístico SPSS, licenciado por la Fundación Universitaria del Areandina, realizando un primer análisis descriptivo con determinación de la normalidad de los datos con la prueba Shapiro-Wilk con un posterior análisis inferencial para la determinación de diferencias en las variables sociodemográficas con el estadístico $\mathrm{T}$ de Student. Igualmente, se determinaron las diferencias de la fuerza muscular isométrica entre el género masculino y femenino y entre el miembro superior dominante y no dominante, utilizando para ello el estadístico T de student. Posteriormente se efectuó un análisis de varianza (ANOVA) de dos factores para medidas repetidas para analizar el efecto del género en la fuerza muscular de cada uno de los músculos evaluados.

\section{RESULTADOS}

19 nadadores del Club Flamingo de Natación de Risaralda participaron en este estudio, de los cuales 9 fueron hombres y 9 fueron mujeres con una media para la edad de $18 \pm 4,1$ y 17,1 $\pm 4,3$ años respectivamente, una media para el índice de masa corporal en hombres de $22,8 \pm 2 \mathrm{Kg} / \mathrm{m} 2$ y en mujeres de $22,2 \pm 1,1, \mathrm{Kg} / \mathrm{m} 2$ mientras que el porcentaje graso mostró una media de $18,2 \% \pm 6,2$ en hombres y de $29,9 \% \pm$ 3,2 en mujeres. No se evidenciaron diferencias estadísticamente significativas entre el género masculino y femenino para estas variables demográficas a excepción del peso, el cual arrojó una $\mathrm{p}<0,05$. Estas variables se presentan en la tabla 1.

Tabla 1.

Variables Sociodemográficas de los participantes.

\begin{tabular}{cccc}
\hline Variable & Género Masculino & Género Femenino & \\
\cline { 2 - 3 } & $\mathbf{n}=\mathbf{9}$ & $\mathbf{n}=\mathbf{9}$ & \\
\hline Edad & $18 \pm 4,1$ & $17,1 \pm 4,3$ & 0,906 \\
Peso $(\mathrm{Kg})$ & $69,4 \pm 10,9$ & $58,2 \pm 3,9$ & $0,024^{*}$ \\
Talla (cm) & $174 \pm 7,9$ & $163 \pm 4,1$ & 0,099 \\
IMC (Kg/m2) & $22,8 \pm 2$ & $22,2 \pm 1,1$ & 0,063 \\
\% Graso & $18,2 \pm 6,2$ & $29,9 \pm 3,2$ & 0,31 \\
\hline
\end{tabular}


Al comparar la fuerza muscular isométrica de los músculos rotadores externos e internos de la articulación glenohumeral entre el miembro superior dominante y no dominante, no se encontraron diferencias estadísticamente significativas ni en los hombres ni en las mujeres $(\mathrm{p}>0,05)$. Tampoco se encontraron diferencias significativas en la fuerza muscular isométrica de los músculos implicados en el control de la cintura escapular entre el miembro inferior dominante y no dominante, tanto para el género masculino como para el femenino $(\mathrm{p}>0,05)$. Estos datos se presentan en la tabla 2.

\section{Tabla 2.}

Fuerza muscular isométrica de la musculatura rotadora de la articulación glenohumeral $y$ de la musculatura de la cintura escapular en miembro superior dominante y no dominante.

\begin{tabular}{lcccccc}
\hline \multirow{2}{*}{ Variable } & \multicolumn{3}{c}{ Género Masculino } & \multicolumn{2}{c}{ Género Femenino } & \\
\cline { 3 - 6 } & Dominante & No Dominante & p valor & No Dominante & Dominante & p valor \\
\hline Rotadores Externos & $140,1 \pm 28$ & $130,6 \pm 23,4$ & 0,09 & $98,1 \pm 25,5$ & $94,3 \pm 15,3$ & 0,52 \\
Rotadores Internos & $137,4 \pm 23,9$ & $136,8 \pm 21,1$ & 0,9 & $100,6 \pm 25,4$ & $99,6 \pm 18,9$ & 0,82 \\
Trapecio Superior & $396,8 \pm 79,9$ & $389,3 \pm 61,9$ & 0,4 & $346,6 \pm 44,9$ & $348,5 \pm 39,8$ & 0,66 \\
Trapecio Medio & $186,1 \pm 35,5$ & $187,7 \pm 26,7$ & 0,71 & $163,9 \pm 27,3$ & $170,7 \pm 28,7$ & 0,17 \\
Trapecio Inferior & $220,6 \pm 41,7$ & $223,2 \pm 37,7$ & 0,46 & $166,4 \pm 26,2$ & $180,2 \pm 31,4$ & 0,06 \\
Romboides & $183,9 \pm 34,7$ & $175,9 \pm 29,9$ & 0,24 & $138,6 \pm 19$ & $134,1 \pm 26,8$ & 0,61 \\
Serrato Anterior & $248,5 \pm 47,9$ & $234,4 \pm 39,7$ & 0,12 & $174 \pm 24,6$ & $171,1 \pm 30,6$ & 0,61 \\
\hline
\end{tabular}

El balance de la fuerza muscular isométrica tanto para los músculos rotadores de la articulación glenohumeral como para los músculos encargados del control de la cintura escapular, tampoco mostraron diferencias estadísticamente significativas entre el miembro superior dominante y el no dominante, tanto en hombres como en mujeres en los nadadores del Club Flamingo de la Liga de Risaralda.

Los datos correspondientes al balance de fuerza muscular se presentan en la tabla 3. Se encontraron diferencias estadísticamente significativas entre la fuerza muscular del trapecio inferior, serrato anterior, romboides, rotadores externos y rotadores internos del hombro tanto en hombres como en mujeres $(\mathrm{p}<0,05)$. No se encontraron diferencias estadísticamente significativas entre la fuerza del músculo trapecio superior y trapecio medio ni en hombres ni en mujeres $(p>0,05)$. 
Tabla 3.

Cocientes de fuerza muscular isométrica en la musculatura rotadora de la articulación glenohumeral y en cintura escapular.

\begin{tabular}{lcccccc}
\hline Variable & & Género Masculino & \multicolumn{2}{c}{ Género Femenino } & \\
\cline { 3 - 5 } & Dominante & No Dominante & p valor & Dominante & No Dominante & p valor \\
\hline $\begin{array}{c}\text { Rotadores } \\
\text { externos/rotadores } \\
\text { internos }\end{array}$ & $1,03 \pm 0,17$ & $0,96 \pm 0,14$ & 0,28 & $0,98 \pm 0,12$ & $0,95 \pm 0,08$ & 0,35 \\
$\begin{array}{l}\text { Trapecio superior/ } \\
\text { trapecio inferior }\end{array}$ & $1,82 \pm 0,35$ & $1,77 \pm 0,31$ & 0,2 & $2,12 \pm 0,39$ & $1,97 \pm 0,34$ & 0,89 \\
$\begin{array}{l}\text { Serrato anterior/ } \\
\text { trapecio medio }\end{array}$ & $1,36 \pm 0,30$ & $1,25 \pm 0,18$ & 0,94 & $1,08 \pm 0,18$ & $1,01 \pm 0,17$ & 0,22 \\
$\begin{array}{l}\text { Serrato anterior/ } \\
\text { romboides }\end{array}$ & $1,36 \pm 0,17$ & $1,33 \pm 0,12$ & 0,78 & $1,26 \pm 0,21$ & $1,29 \pm 0,21$ & 0,7 \\
\hline
\end{tabular}

\section{DISCUSIÓN}

Dentro de los principales hallazgos de esta investigación, se encuentra la inexistencia de diferencias estadísticamente significativas en la fuerza muscular isométrica del hombro entre el miembro superior dominante y no dominante, tanto en el género masculino como en el género femenino. Resultados similares reportaron Riemann et al. (7) quienes no encontraron diferencias significativas en la fuerza muscular isométrica de los rotadores externos del hombro entre el miembro superior dominante y no dominante. Sin embargo, al contrario de la presente investigación, sí encontraron diferencias estadísticamente significativas en la fuerza de los músculos rotadores internos del hombro entre el miembro superior dominante y no dominante.

Meliscki et al. (8) también mostraron asimetrías en la musculatura del hombro entre el miembro superior dominante y el no dominante en nadadores de élite. Es posible que el grado de entrenamiento de nadadores de élite marque diferencia en torno a las adaptaciones crónicas que sufre la musculatura, mostrando asimetrías marcadas en este tipo de deportistas, en comparación a atletas menos experimentados como los del presente estudio.

En cuanto al coeficiente de fuerza muscular isométrica de los rotadores internos y externos de la articulación glenohumeral, tampoco se evidenció una diferencia significativa respecto al género y a la dominancia de los miembros superiores. De igual forma, tampoco se encontraron diferencias significativas en el cociente de la fuerza muscular isométrica entre el músculo trapecio superior en comparación al trapecio inferior, como del músculo serrato anterior en comparación al trapecio medio 
y el romboides. Turner et al. (3), aunque reportaron los valores de estos cocientes, los cuales fueron superiores a los reportados en esta investigación, no determinaron diferencias por género ni por dominancia lateral.

Estos mismos investigadores, (3) reportaron diferencias significativas entre la fuerza de cada músculo, indicando un mayor nivel de fuerza muscular para el trapecio superior, seguido del serrato anterior, el trapecio medio, romboides y finalmente el trapecio inferior. En esta investigación se encontró un orden de fuerza muscular diferente tanto para el género masculino como para el género femenino, obteniéndose un mayor nivel de fuerza para el trapecio superior, seguido del serrato anterior, trapecio inferior, trapecio medio y finalmente el romboides. Los menores niveles de fuerza fueron para los rotadores externos e internos del hombro, siendo los del género masculino más fuertes que los del género femenino.

La mayor limitante de este estudio fue el tamaño de la muestra, sin embargo, es importante dimensionar la relevancia de los sujetos involucrados, dado que correspondían a nadadores y nadadoras jóvenes, en los cuales la detección temprana de imbalances y asimetrías de la fuerza isométrica de la musculatura del hombro pueden significar grandes cambios para la prevención de lesiones y el aumento del rendimiento deportivo.

En el futuro, al caracterizar otras poblaciones, se deben considerar variables como la movilidad articular y la estática escapular, para así poder tener comparaciones y asociaciones que posibiliten completar un mejor perfil de riesgo músculo-esquelético para la prevención de lesiones de hombro en nadadores de la región.

\section{CONCLUSIONES}

Los hallazgos de la presente investigación permiten concluir que en un deporte en el cual se requieren ambos miembros superiores como lo es la natación, no hay una diferencia significativa en cuanto a la fuerza isométrica de la musculatura del hombro con respecto al miembro superior dominante y no dominante. Además, se pudo establecer que no se presentan diferencias en el balance y simetría de la musculatura del hombro tanto en el género masculino como en el género femenino. 
1. Habechian FAP, Van Malderen K, Camargo PR, Cools AM. Changes in shoulder girdle strength in 3 consecutive years in elite adolescent swimmers: a longitudinal cohort study. Brazilian J Phys Ther. 2018;22(3):238-47.

2. Linklater J, Appleyard R, Kirkbride B, Walton J, Sein ML, Murrell GAC, et al. Shoulder pain in elite swimmers: primarily due to swim-volume-induced supraspinatus tendinopathy. Br J Sports Med. 2008;44(2):105-13.

3. Turner N, Ferguson K, Mobley BW, Riemann B, Davies G. Establishing Normative Data on Scapulothoracic Musculature Using Handheld Dynamometry. 2009;502-20.

4. Johansson FR, Skillgate E, Lapauw ML, Clijmans D, Deneulin VP, Palmans $\mathrm{T}$, et al. Measuring eccentric strength of the shoulder external rotators using a handheld dynamometer: Reliability and validity. J Athl Train. 2015;50(7):719_ 25 .

5. Hill L, Collins M, Posthumus M. Risk factors for shoulder pain and injury in swimmers : A critical systematic review Risk factors for shoulder pain and injury in swimmers : A critical systematic review. 2015;384.

6. Hislop H, Montgomery J. Daniels and Worthingham's Muscle Testing: Techniques of Manual Examination. 7th ed. Philadelphia, PA: Elsevier Science; 2002.

7. Riemann BL, Davies GJ, Ludwig L, Gardenhour H. Hand-held dynamometer testing of the internal and external rotator musculature based on selected positions to establish normative data and unilateral ratios. J Shoulder Elb Surg [Internet]. 2010;19(8):1175-83.

8. Meliscki, Gustavo Antonio, et al. Alterations in strength of the shoulder rotators in young elite swimmers. Fisioterapia en Movimento. 30.1 (2017): $11-18$. 


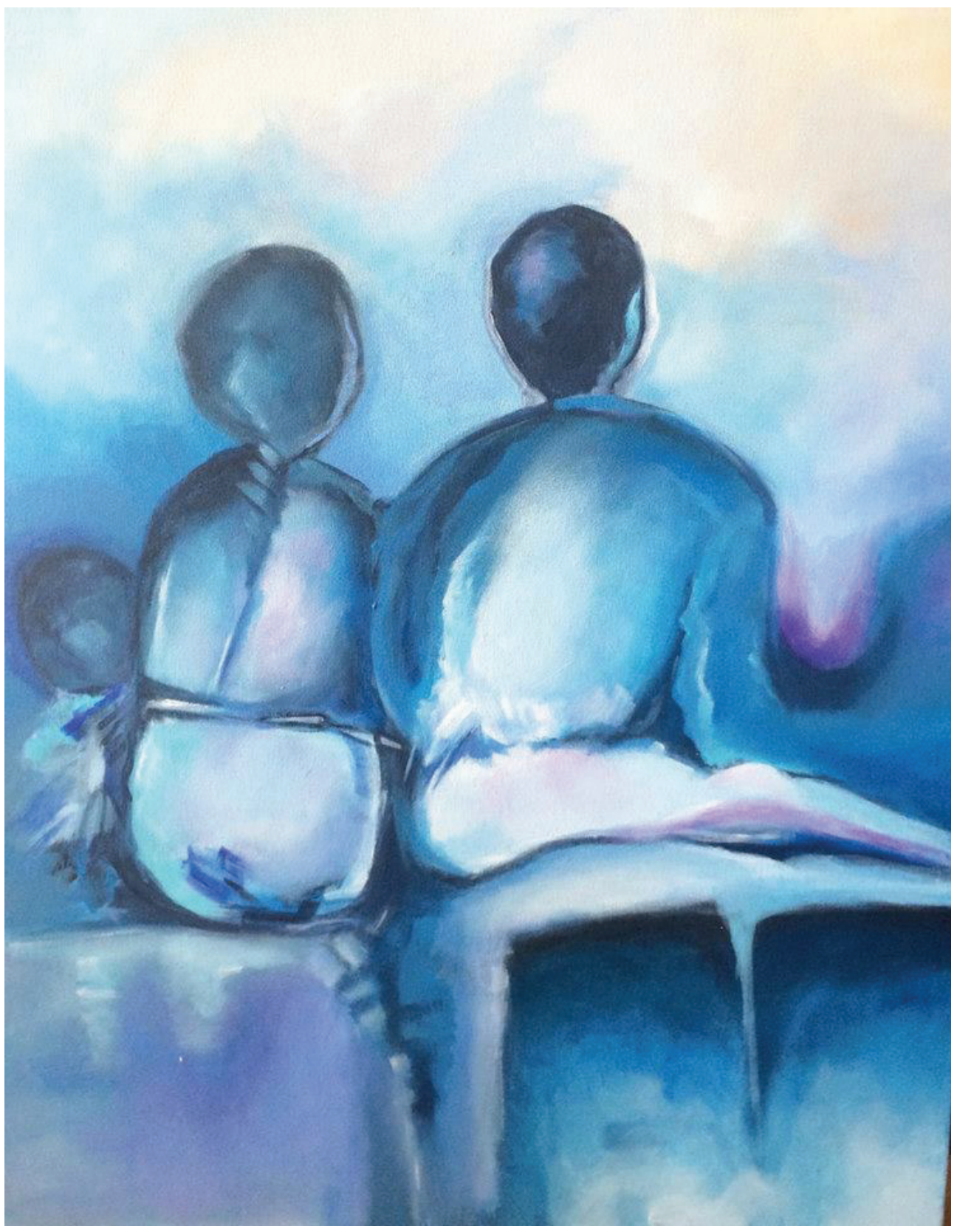

Acrílico sobre lienzo. "La Espera”, Virginia Aristizábal Parra. 